\title{
Orange-colored diapers as first sign of Lesch-Nyhan disease in an asymptomatic infant
}

\author{
Serena Gasperini • Stefano Stagi • Ugo Gasperini • \\ Renzo Guerrini • Giancarlo la Marca • \\ Maria Alice Donati
}

Received: 21 April 2010 / Accepted: 22 April 2010

(C) IPNA 2010

Sirs,

We read with great interest the letter of Demirdas and Schröder [1] about the differential diagnosis in changed color of urine in infants.

We have followed a 3-month-old male who was hospitalized for the presence of orange-colored diapers. He was born from unrelated parents after an uneventful pregnancy. High levels of uric acid were found in the plasma $(11.7 \mathrm{mg} / \mathrm{dl}$; normal values $1.7-5.8)$. Kidney ultrasound examination showed mild and diffuse increase of signal in cortical area with nephrolithiasis. Fundus oculi, electroencephalogram (EEG), and brain ultrasound were normal. Successively, the infant presented dystonic movements, mild axial hypotonia, and hypertonia of the legs with increased tendon reflexes and scissoring. The study of purine and pyrimidine metabolism by electrospray ionization tandem mass spectrometry (MS-MS) showed high

S. Gasperini · R. Guerrini - G. la Marca • M. A. Donati

Department of Pharmacology, University of Florence,

Florence, Italy

S. Stagi

Pediatric Unit, Villamarina Hospital,

Piombino, Livorno, Italy

U. Gasperini

Paediatric Unit-“Ceppo” Hospital Pistoia,

Pistoia, Tuscany, Italy

S. Gasperini $(\bowtie)$

Metabolic Diseases Unit, Meyer Children Hospital of Florence,

Viale Pieraccini 24,

50139 Florence, Italy

e-mail: s.gasperini@meyer.it levels of hypoxanthine $(20.3 \mathrm{mmol} / \mathrm{mol}$ of creatinine; normal values 0.43-11.31). Enzymatic assay in red cells showed an absence of hypoxanthine-guanine phosphoribosyltransferase activity (HGPRT). Molecular analysis confirmed the diagnosis of Lesch-Nyhan disease (LND; OMIM 300322) in the patient and his mother as a carrier: a duplication of 8 nucleotides 89-96 (89_96dupAGGATTTG) in exon 2 of HPRT1 gene was found in chromosome X.

Brain magnetic resonance imaging showed white matter atrophy of bilateral symmetric temporal-insular regions with respective enlargement of subarachnoid spaces with mild decreased concentration of NAA and Cho at spectroscopy. At 20 months, the patient was unable to control his head or speak; he also showed swallowing and severe dystonic-dyskinetic movement disorder of the upper limbs with generalized hypertonia crisis towards opistotonus.

LND is a rare X-linked recessive disorder [2] characterized by the progressive development of mental retardation, spasticity, choreo-athetosis, self-mutilation, and hyperuricemia [3, 4], due to complete deficiency of the activity of HGPRT (OMIM 308000) [2]. However, partially HGPRTdeficient patients (OMIM 300323) present these symptoms with different degrees of intensity [5].

In these patients, orange crystals may be identified in diapers during the first weeks of life, but only rarely are they an early sign of the disease $[3,4,6]$. For example, Jinnah et al. [7] reported only one patient in 40 with LND.

Orange crystals are often present in diapers during the first weeks of life, when deposits of pink or red uric acid crystals are observable [8]. Urate crystals are orange-red precipitates formed in the bladder, which look like 'brick dust' in a diaper [1]. Notwithstanding, orange-colored urine could be due to a variety of causes. The commonest is due to some food colors such as phenolphthalein or beet, drugs 
such as rifampicin, multivitamins, concentrated urine due to poor water intake, and finally, some urinary bacterial infections as well as hematuria can result in red-colored urine (Citrobacter sedlakii) [1].

In conclusion, we are pleased that our colleagues have discussed this important problem. Our case report seems to suggest that orange-colored diapers after the neonatal period, even if not associated with evident neurological and renal impairment, may be due to this metabolic disorder.

\section{References}

1. Demirdas S, Schröder CH (2010) An infant with orange-colored urine. Pediatr Nephrol 25:381

2. Jinnah HA (2009) Lesch-Nyhan disease: from mechanism to model and back again. Dis Model Mech 2:116-121
3. Torres RJ, Puig JG (2007) Hypoxanthine-guanine phosphoribosyltransferase (HPRT) deficiency: Lesch-Nyhan syndrome. Orphanet J Rare Dis 2:48

4. Jinnah HA, De Gregorio L, Harris JC, Nyhan WL, O'Neill JP (2000) The spectrum of inherited mutations causing HPRT deficiency: 75 new cases and a review of 196 previously reported cases. Mutat Res 463:309-326

5. García MG, Torres RJ, Prior C, Puig JG (2008) Normal HPRT coding region in complete and partial HPRT deficiency. Mol Genet Metab 94:167-172

6. Yang MT, Mak SC, Chi CS, Lin HY, Lii YP, Wu KH, Shian WJ (1994) Lesch-Nyhan Syndrome: report on two brothers. Zhonghua Min Guo Xiao Er Ke Yi Xue Hui Za Zhi 35:552-558

7. Jinnah HA, Visser JE, Harris JC, Verdu A, Larovere L, CeballosPicot I, Gonzalez-Alegre P, Neychev V, Torres RJ, Dulac O, Desguerre I, Schretlen DJ, Robey KL, Barabas G, Bloem BR, Nyhan W, De Kremer R, Eddey GE, Puig JG, Reich SG, LeschNyhan Disease International Study Group (2006) Delineation of the motor disorder of Lesch-Nyhan disease. Brain 129:1201-1217

8. Küpeli S, Yurdakök M, Kilinc G, Bilgetekin E (2005) Urinary uric acid levels and discoloration of diapers in healthy neonates. Pediatr Nephrol 20:1361-1362 